\title{
Mitochondrial leucine tRNA level and PTCD1 are regulated in response to leucine starvation
}

\author{
Christof Schild • Dagmar Hahn • André Schaller • \\ Christopher Benjamin Jackson • Barbara Rothen-Rutishauser • \\ Jelena Mirkovitch $\cdot$ Jean-Marc Nuoffer
}

Received: 3 February 2014/ Accepted: 17 March 2014/Published online: 8 April 2014

(C) Springer-Verlag Wien 2014

\begin{abstract}
Pentatricopeptide repeat domain protein 1 (PTCD1) is a novel human protein that was recently shown to decrease the levels of mitochondrial leucine tRNAs. The physiological role of this regulation, however, remains unclear. Here we show that amino acid starvation by leucine deprivation significantly increased the mRNA steadystate levels of PTCD1 in human hepatocarcinoma (HepG2) cells. Amino acid starvation also increased the mitochondrially encoded leucine tRNA (tRNA ${ }^{\mathrm{Leu}(\mathrm{CUN})}$ ) and the mRNA for the mitochondrial leucyl-tRNA synthetase (LARS2). Despite increased PTCD1 mRNA steady-state levels, amino acid starvation decreased PTCD1 on the protein level. Decreasing PTCD1 protein concentration increases the stability of the mitochondrial leucine tRNAs, tRNA $^{\text {Leu(CUN) }}$ and tRNA ${ }^{\text {Leu(UUR) }}$ as could be shown by RNAi experiments against PTCD1. Therefore, it is likely that decreased PTCD1 protein contributes to the increased tRNA $^{\text {Leu(CUN) }}$ levels in amino acid-starved cells. The stabilisation of the mitochondrial leucine tRNAs and the upregulation of the mitochondrial leucyl-tRNA synthetase $L A R S 2$ might play a role in adaptation of mitochondria to amino acid starvation.
\end{abstract}

C. Schild $(\bowtie) \cdot$ D. Hahn · C. B. Jackson · J. Mirkovitch ·

J.-M. Nuoffer

Institute of Clinical Chemistry, Inselspital, Bern University

Hospital and University of Bern, Bern, Switzerland

e-mail: christof.schild@insel.ch

\section{A. Schaller}

Division of Human Genetics, Department of Pediatrics, Bern

University Hospital and University of Bern, Bern, Switzerland

B. Rothen-Rutishauser

Adolphe Merkle Institute, University of Fribourg,

Fribourg, Switzerland
Keywords Amino acid deprivation - Mitochondrion · tRNA · Pentatricopeptide repeat (PPR) protein . Pentatricopeptide repeat domain protein 1 (PTCD1) . RNA stability

\section{Introduction}

Homeostasis of amino acids and proteins is maintained by the regulation of protein synthesis and proteolysis, de novo synthesis and degradation of amino acids and nutritional intake (Chaveroux et al. 2010). Amino acid availability regulates nuclear gene expression on the level of transcription, mRNA stability, translation, and protein degradation (Siu et al. 2002; Harding et al. 2003; Averous et al. 2004; Leung-Pineda et al. 2004; Chaveroux et al. 2010).

Amino acid availability was also shown to influence the expression of mitochondrial genes and mitochondrial metabolism. Leucine starvation induces the translation of the branched chain $\alpha$-keto acid dehydrogenase kinase and in this way suppresses the degradation of branched chain amino acids in mitochondria (Doering and Danner 2000; Harris et al. 2001). Cultivation of cells in high leucine concentration increases mitochondrial mass, expression of mitochondrial proteins and mitochondrial respiration (Sun and Zemel 2009). While amino acid starvation induces a rapid degradation of cytosolic proteins by autophagy; mitochondrial proteins are much more stable because functional mitochondria are protected against mitophagy (Kristensen et al. 2008; Rambold et al. 2011).

Recently, the pentatricopeptide repeat domain protein 1 (PTCD1), a nuclear-encoded mitochondrial protein was shown to be involved in mitochondrial RNA processing and in the reduction of steady-state levels of mitochondrial leucine tRNAs. Lowering PTCD1 in cells increased the 
levels of several mitochondria-encoded proteins and the activity of complex IV of the respiratory chain (Rackham et al. 2009; Sanchez et al. 2011). PTCD1 was also shown to bind to the mitochondrial RNase ELAC2 and to affect the $3^{\prime}$-processing of mitochondrial tRNAs. It is not yet clear, how the effect of PTCD1 as a negative regulator of mitochondrial leucine tRNAs and the effect of PTCD1 in 3'processing are related (Rackham and Filipovska 2012).

Targeted destruction of cytoplasmic tRNA is a conserved response to oxidative stress in mammalian cells, yeast, and plants (Thompson et al. 2008; Yamasaki et al. 2009). In yeast, methionine starvation was shown to induce degradation of cytoplasmic tRNA (Thompson et al. 2008). The physiological role of decreased mitochondrial tRNA level by PTCD1, however, remains to be discovered. Here, we show that both mitochondrial tRNA levels and PTCD1 protein levels are regulated in response to amino acid starvation. PTCD1 might, therefore, be involved in the regulation of mitochondrial translation in response to amino acid starvation.

\section{Materials and methods}

\section{Cell culture}

Cells were cultivated in minimal essential medium (MEM) supplemented with $10 \%$ foetal calf serum, $1 \times$ nonessential amino acids (11140-35, Gibco/Invitrogen, Carlsbad, CA), $2 \mathrm{mM}$ L-glutamine, $200 \mu \mathrm{M}$ uridine, $1 \mathrm{mM}$ sodium pyruvate, $100 \mathrm{U} / \mathrm{ml}$ penicillin, and $100 \mu \mathrm{g} / \mathrm{ml}$ streptomycin and $10 \mu \mathrm{g} / \mathrm{ml}$ chlortetracycline at $37{ }^{\circ} \mathrm{C}$ and $5 \% \mathrm{CO}_{2}$. For the cultivation in different L-leucine concentrations, the above medium but without L-leucine was supplemented with the indicated L-leucine concentrations and dialysed instead of normal foetal calf serum.

\section{RNA extraction and quantification}

Total RNA was isolated using the miRNeasy Kit including DNase treatment (Qiagen GmbH, Hilden, Germany). $1 \mu \mathrm{g}$ RNA together with random hexamers was denatured at $65{ }^{\circ} \mathrm{C}$, cooled on ice and then transcribed into firststrand cDNA by Thermoscript reverse transcriptase as suggested by the supplier (Invitrogen). Following transcription, the enzyme was heat inactivated at $85^{\circ} \mathrm{C}$. The cDNA was quantified by real-time PCR with an ABI 7500 Sequence Detection System utilising the Power SYBR Green PCR master mix (Applied Biosystems). A minus reverse transcriptase control was included to control DNase treatment. Primers used are listed in Table 1. Annealing temperature was $60{ }^{\circ} \mathrm{C}$. All experiments were performed in triplicates.
To determine the RNA degradation rate, de novo synthesis of mitochondrial RNA was selectively inhibited with $\mathrm{EtBr}$ (Yasukawa et al. 2000). $250 \mathrm{ng} / \mathrm{ml}$ was the lowest concentration that showed maximal inhibition in HepG2 cells. The degradation rate of mitochondrial RNAs was observed by real-time PCR.

\section{RNA interference}

For RNA interference-mediated downregulation a modified pSuper vector containing a puromycin-resistance was used (Paillusson et al. 2005). Sequences coding for short hairpin RNAs were ligated as double-stranded oligonucleotides into the vector. Oligonucleotides for PTCD1 knockdown construct: 5'-GAT CCC CTC TAC AGT GCC CTC ATC AAT TCA AGA GAT TGA TGA GGG CAC TGT AGA TTT TTA- ${ }^{\prime}$ and $5^{\prime}$-AGC TTA AAA ATC TAC AGT GCC CTC ATC AAT CTC TTG AAT TGA TGA GGG CAC TGT AGA GGG-3' ${ }^{\prime}$, the 19 nucleotide sequence specific stretch is underlined. As a control, sequences coding for a non-targeted control (OligoEngine) were inserted likewise: 5'-GAT CCC CGC GCG CTT TGT AGG ATT CGT TCA AGA GAC GAA TCC TAC AAA GCG CGC TTT TTA- ${ }^{\prime}$ and $5^{\prime}$ AGC TTA AAA AGC GCG CTT TGT AGG ATT CGT CTC TTG AAC GAA TCC TAC AAA GCG CGC GGG-3'. Vector constructs containing PTCD1 knockdown construct or non-targeted constructs were linearised by ScaI digestion. HepG2 cells were transfected either with linearised PTCD1 knockdown construct or linearised non-targeted construct and selected with $1 \mu \mathrm{g} / \mathrm{ml}$ puromycin for 10 days. Healthy colonies were picked with cloning cylinders and screened for PTCD1 knockdown by real-time PCR. Before analysis, the cells were cultivated without puromycin for at least $16 \mathrm{~h}$.

\section{Mitochondrial isolation and respiratory chain analysis}

Isolation of mitochondria and activity measurements of citrate synthase (CS), NADH-ubiquinone oxidoreductase (CI), succinate-ubiquinone oxidoreductase (CII), ubiquinol-cytochrome c reductase (CIII), cytochrome c oxidase (CIV) and ATP synthase (CV) were determined spectrophotometrically as described previously (Schaller et al. 2011; Jackson et al. 2014).

\section{Western blots}

Exponentially growing cultures of HepG2 cells were briefly rinsed twice with phosphate-buffered saline and dissolved in RIPA lysis buffer containing PMSF, sodium orthovanadate and protease inhibitor cocktail (sc-24948, Santa Cruz Biotechnology). 20- $\mu$ g protein was combined with SDS sample buffer containing dithiothreitol. Proteins were resolved on SDS-polyacrylamide gels according to 
Table 1 Oligonucleotide sequences

\begin{tabular}{|c|c|c|c|}
\hline Name & Fwd primer $\left(5^{\prime}-3^{\prime}\right)$ & Rev primer $\left(5^{\prime}-3^{\prime}\right)$ & Amplicon (bp) \\
\hline PTCD1 F & CGTGGTCACAGAGGAGACCT & & 116 \\
\hline PTCD1 R & & GCTGTAGCCCTAGACTCAGCA & \\
\hline$\beta$-Actin F & GCCAACCGCGAGAAGATG & & 98 \\
\hline$\beta$-Actin R & & CCAGAGGCGTACAGGGATAG & \\
\hline GAPDH F & CATTGACCTCAACTACATGGTTTAC & & 136 \\
\hline GAPDH R & & GGGATCTCGCTCCTGGAAG & \\
\hline tRNA $^{\text {Leu(CUN)a }}$ & AATAAATCATAAATCCATTGGTCTTAGGCCCC & & 74 \\
\hline tRNA $^{\text {Leu(CUN)a }}$ & & AATAAATCATAAACTTTTATTTGGAGTTGCACCA & \\
\hline tRNA $^{\text {Leu(UUR)a }}$ & AATAAATCATAAGGCAGAGCCCGGTAATCGCA & & 91 \\
\hline tRNA $^{\text {Leu(UUR)a }}$ & & AATAAATCATAATGTTAAGAAGAGGAATTGAAC & \\
\hline LARS1 F & TTTCCCGCCACTGGCTATTC & & 178 \\
\hline LARS1 R & & CGGAGAGTAAATTGTATACCGCTTC & \\
\hline LARS2 F & TCTCAGGTGACCACCCATTTC & & 141 \\
\hline LARS2 R & & GGCACACAAAGCATCCTCAAAC & \\
\hline ND3 $F^{a}$ & ACCACAACTCAACGGCTACA & & 169 \\
\hline ND3 $R^{a}$ & & TTGTAGGGCTCATGGTAGGG & \\
\hline ATF4 F & CCAACAACAGCAAGGAGGATG & & 92 \\
\hline ATF4 R & & ACCCAACAGGGCATCCAAG & \\
\hline
\end{tabular}

${ }^{a}$ Adapted from Rackham et al. (2009)

the method of Laemmli, and transferred to polyvinylidene difluoride membranes by electroblotting. Unspecific sites were blocked with Tris-buffered saline (TBS) contain $5 \%$ non-fat dried milk and $0.1 \%$ Tween-20. The blots were incubated with antibodies against human PTCD1 (SigmaAldrich, product no. HPA020106) diluted 1:2,000 in TBS containing $1 \%$ non-fat dried milk, $0.1 \%$ Tween-20.

The signal was detected using a chemiluminescence ECL detection kit (Amersharm Biosciences, Inc.) with horseradish peroxidase-conjugated secondary antibodies and lumigen PS-3 acridan as a substrate using Chemidoc detection system (Bio-Rad). The membranes were stripped with $0.2 \mathrm{M} \mathrm{NaOH}$ and reprobed with a monoclonal antibody against human porin in a dilution of 1:3,300 (MitoSciences, product no. MSA03).

Laser scanning microscopy

Human full-length PTCD1 but lacking the stop codon was cloned into a pEGFP-N2 vector (Clontech, Palo Alto, CA, USA) in frame with the EGFP sequence. Correct sequence of the PTCD1-EGFP open reading frame was verified by sequencing. HepG2 cells were seeded onto borosilicatechambered cover glasses (Nunc No. 155380) at a density of 24,000 cells $/ \mathrm{cm}^{2}$. After 2 days, cells were transfected with PTCD1-EGFP plasmid DNA or control EGFP plasmid DNA using FuGENE HD (Roche applied science, Switzerland). After 1-day post-transfection, cells were incubated with $10 \mathrm{nM}$ MitoTracker RedCMXRos (Invitrogen Molecular probes, Eugene, OR) and $1 \mu \mathrm{g} / \mathrm{ml}$ Hoechst
33342 (Invitrogen Molecular probes, Eugene, OR) for 10 min. A laser scanning microscope (Zeiss LSM 510 Meta on Axiovert $200 \mathrm{M}$ inverted microscope; Zeiss, Jena, Germany) was used to visualise the distribution of fluorescence.

\section{Results}

The influence of leucine starvation on the steady-state levels of PTCD1 mRNA, PTCD1 protein and on the steady-state levels of the mitochondrial leucine tRNAs was investigated in human hepatocarcinoma cells (HepG2). For this, HepG2 cells were cultivated for $48 \mathrm{~h}$ in a modified minimal essential medium (MEM) containing either the original L-leucine concentration of MEM $(52 \mathrm{mg} / \mathrm{L} \mathrm{L-leu-}$ cine) or reduced leucine concentrations (0, 0.52 or $5.2 \mathrm{mg} / \mathrm{l}$ L-leucine). As shown in Fig. 1a, leucine starvation significantly increased the mRNA steady-state level of PTCD1, whereas the level of a cytoplasmic control mRNA GAPDH and a mitochondrial control mRNA ND3 remained constant (Fig. 1g, h). The increase in PTCDl mRNA was similar to the increase of the activating transcription factor 4 mRNA (ATF4) (Fig. 1b). ATF4 is known to be induced on mRNA and protein level by amino acid starvation, serving as a positive control (Siu et al. 2002; Lee et al. 2008). Leucine deprivation also increased the steady-state levels of mRNAs encoding for the cytoplasmic leucyltRNA synthetase (LARS1) and the mitochondrial leucyltRNA synthetase (LARS2) (Fig. 1c, d). One of the two 
Fig. 1 Leucine deprivation increases steady-state levels of PTCD1 mRNA and mitochondrial tRNA ${ }^{\text {Leu(CUN) }}$. HepG2 wild-type cells were cultivated for $48 \mathrm{~h}$ in a medium containing $0,0.52,5.2$ or $52 \mathrm{mg} / \mathrm{l}$ L-leucine. Total RNA was extracted, RNA was reverse transcribed and quantified by quantitative real-time PCR. The quantified RNAs included examples of nuclear-encoded RNAs coding for mitochondrial proteins PTCD1 (a) and LARS2 (d); mitochondrially encoded tRNAs, tRNA ${ }^{\mathrm{Leu}(\mathrm{CUN})}$ (e) and tRNA $^{\text {Leu(UUR) }}$ (f);

mitochondrially encoded mRNA ND3 (g); nuclearencoded RNAs coding for cytoplasmatic proteins ATF4 (b), LARS1 (c) and GAPDH (h). Results were normalised to controls with $52 \mathrm{mg} / \mathrm{l}$ L-leucine and to $\beta$-actin. Results show mean \pm standard error of the mean (SEM) from at least five independent experiments each of which measured in triplicates. ${ }^{*} p<0.05$ by twotailed paired $U$ test
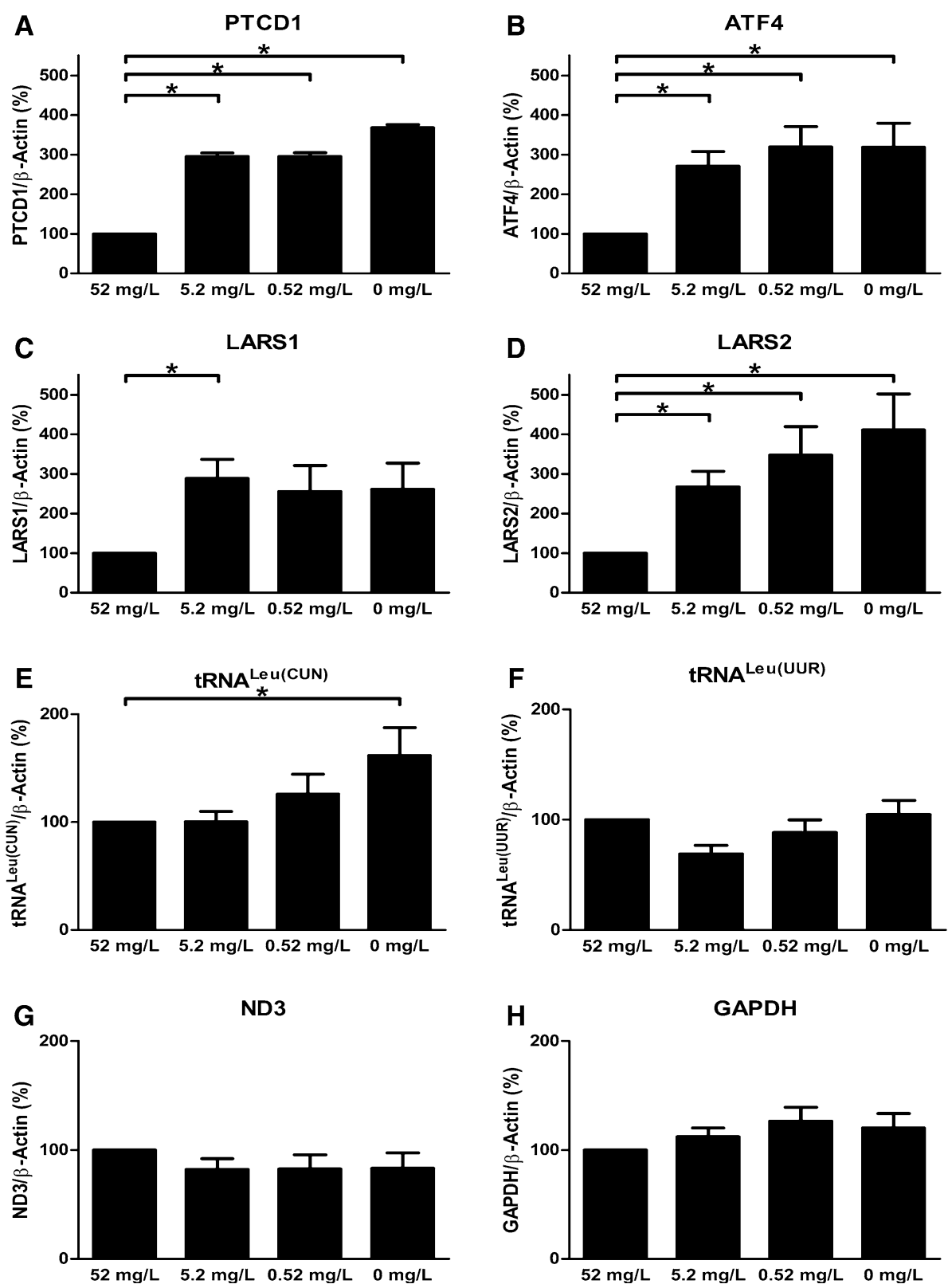

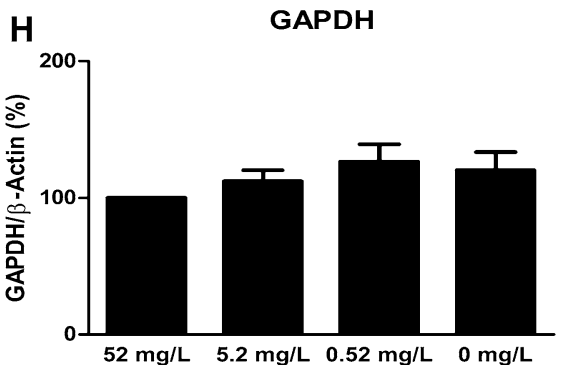

mitochondrial leucine tRNAs, tRNA ${ }^{\text {Leu(CUN) }}$, was significantly increased in cells cultivated without leucine (Fig. 1e). On the other hand, the level of tRNA ${ }^{\text {Leu(UUR) }}$ in cells cultivated without leucine was not significantly different from control cells (Fig. 1f).

To test the effect of leucine starvation at the protein level, Western blot experiments were performed. The antibody against PTCD1 detected a major band with an apparent molecular mass of about $73 \mathrm{kDa}$, which is consistent with the expected mass of human PTCD1 without mitochondrial targeting sequence of $77 \mathrm{kDa}$ (Fig. 2a). Leucine shortage significantly decreased PTCD1 on the protein level within $48 \mathrm{~h}$ (Fig. 2). In tendency ( $p=0.056$ ), this decrease was already observed in cells cultivated in
$5.2 \mathrm{mg} / \mathrm{l}$ leucine - a concentration that permitted normal proliferation for up to $72 \mathrm{~h}$ (data not shown). The decrease of PTCD1 protein in leucine-starved cells despite increased PTCD1 mRNA levels indicates that leucine shortage regulates PTCD1 expression mainly on the translational or post-translational level.

To show that PTCD1 is a mitochondrially targeted protein in HepG2 cells, a GFP-tagged PTCD1 fusion construct was used and visualised by laser scanning microscopy (Fig. 3). These results show, that PTCD1 is indeed co-localised with the mitochondria of HepG2 cells. Therefore, it could act as a potential regulator of mitochondrial tRNA steady-state level. To investigate the effect of PTCD1 downregulation independently from other 
effects of leucine starvation, vectors encoding small interfering RNA (RNAi) against PTCD1 or against a nontargeted sequence were stably transfected into HepG2 cells.
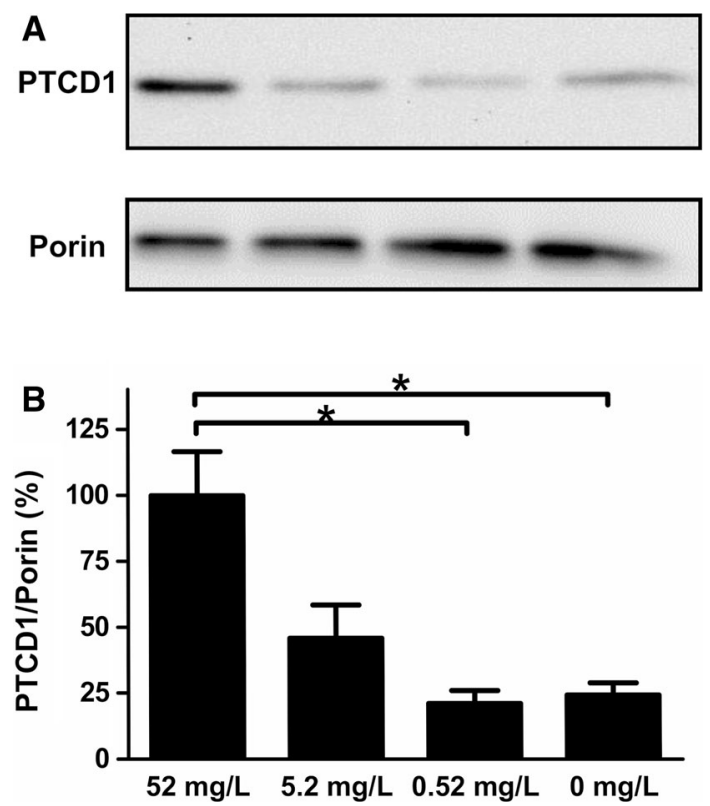

Fig. 2 Leucine deprivation decreases PTCD1 protein steady-state levels. a HepG2 wild-type cells were cultivated for $48 \mathrm{~h}$ in a medium containing $0,0.52,5.2$ or $52 \mathrm{mg} / \mathrm{l}$ L-leucine. b PTCD1 and porin protein was detected by Western blot, and the chemiluminescence signal was quantified. The results represent the mean \pm SEM of four independent experiments. $* p<0.05$ compared to treatment with $52 \mathrm{mg} / \mathrm{l}$ by a by two-tailed $U$ test
These PTCD1 RNAi knockdown cells showed a fourfold decrease in PTCD1 protein, when compared to control cells stably transfected with vector containing a non-targeted RNAi sequence (Fig. 4a). The reduction of PTCD1 in the knockdown cells by RNAi is comparable to the reduction observed in cell cultivated for $48 \mathrm{~h}$ without leucine (Fig. 2). The influence of reduced PTCD1 protein on the degradation rate of leucine tRNAs was then examined by cultivating PTCD1 knockdown cells and control cells in the presence of the mitochondrial transcription inhibitor ethidium bromide (EtBr). Reduction of PTCD1 increased the stability of mitochondrial leucine tRNAs (Fig. 4b). PTCD1 knockdown significantly increased the half-life of tRNA $^{\text {Leu(UUR) }}$ from $4.8 \mathrm{~h}$ [95\% confidence interval (CI) $4.3-5.4 \mathrm{~h}]$ to $13.4 \mathrm{~h}(95 \% \mathrm{CI} 9.3-24.9 \mathrm{~h})$. Stability of tRNA $^{\text {Leu(CUN) }}$ was also increased in PTCD1 knockdown cells, but its half-life could not be determined exactly, because the degradation rate was not constant over time. Stability of mitochondrial mRNA ND3 and ND6 was also increased in PTCD1 knockdown cells (data not shown). These experiments indicate that PTCD1 may be an important factor for the regulation of tRNA ${ }^{\text {Leu }}$ in leucinestarved cells (Fig. 1e).

Next, we assessed the effect of PTCD1 knockdown on the activity of the individual respiratory chain complexes. In our experiments, the activity of complex I was significantly higher in PTCD1 knockdown cells compared to controls transfected with non-targeted RNAi (Fig. 5). In tendency also complex IV activity was increased in our experiments.
Fig. 3 PTCD1 is co-localised with mitochondria. HepG2 hepatocellular carcinoma cells were transfected with PTCD1EGFP fusion construct (upper panel, green signal) or EGFP alone (lower panel, green signal). Mitochondria and nuclei of living cells were stained with mitotracker ( $r e d)$ and Hoechst 33342 (blue), respectively, and observed by laser scanning microscopy. Yellow in the merged image indicates co-localisation of PTCD1-EGFP with mitochondria. Scale bar $10 \mu \mathrm{m}$ (colour figure online)

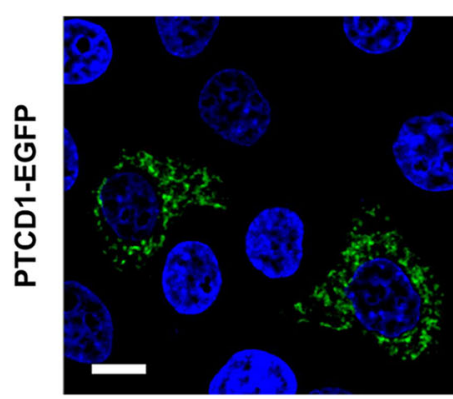

PTCD1-EGFP/Hoechst

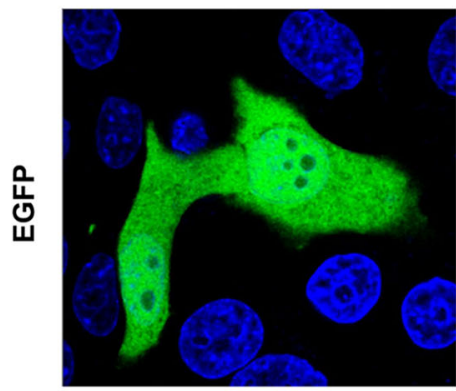

EGFP/Hoechst

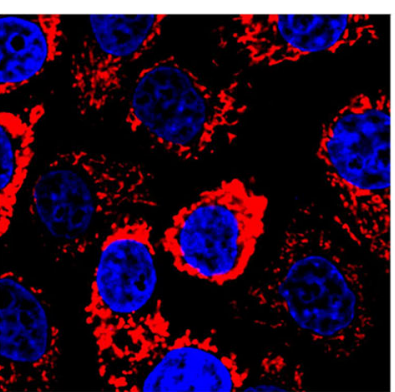

Mitotracker/Hoechst

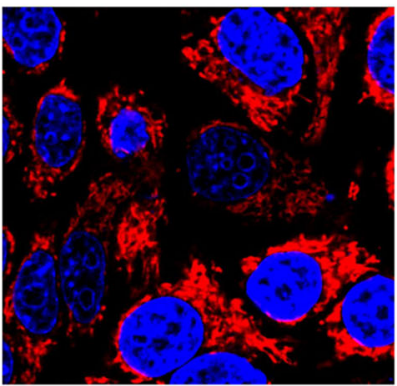

Mitotracker/Hoechst

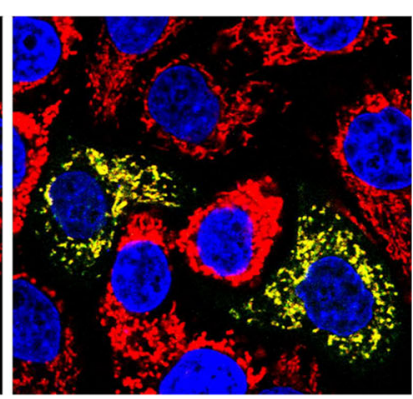

Merged

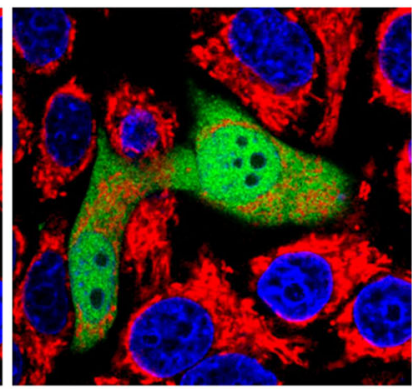

Merged 
A
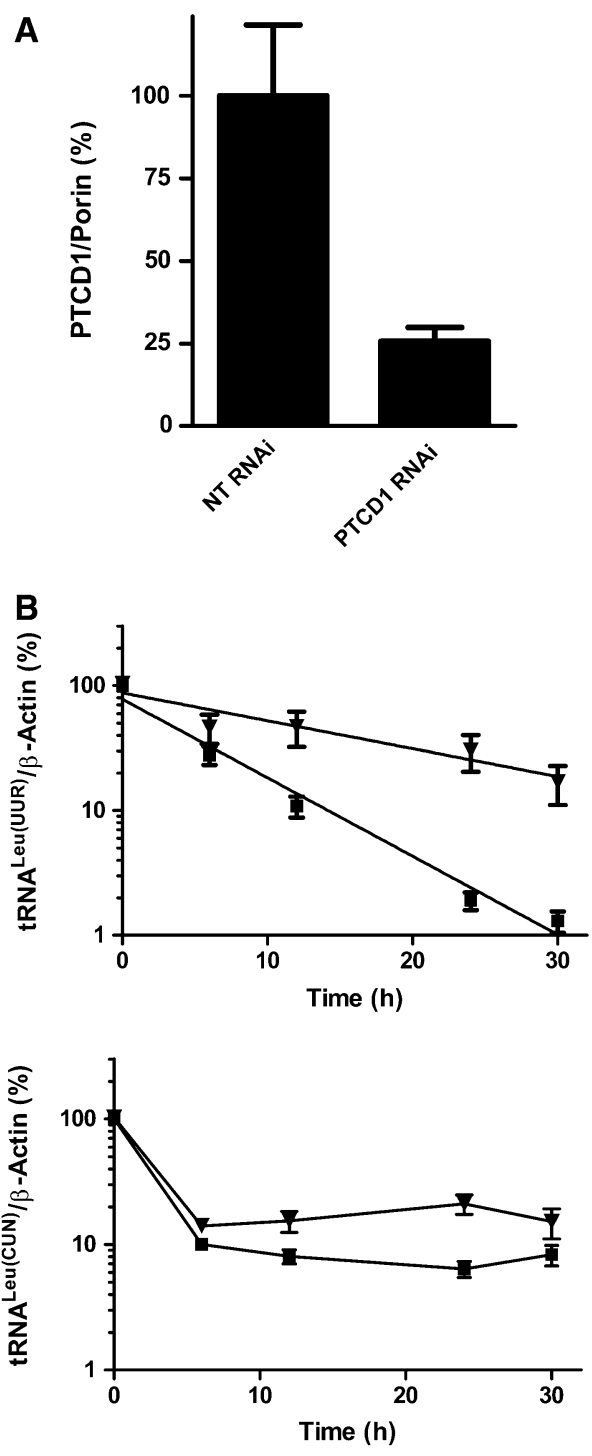

Fig. 4 Stability of mitochondrial RNAs in PTCD1 knockdown cells. a HepG2 cells were stably transfected with small interfering RNAs against PTCD1 (PTCD1 RNAi) or a non-targeted sequence (NT RNAi). PTCD1 and porin protein levels in these PTCD1 knockdown cells and control cells were detected by Western blot, and the chemiluminescence signal was quantified. Mean \pm SEM of four independent experiments. b Stability of mitochondrial leucine tRNAs in cells stably transfected with PTCD1 RNAi (triangles) or nontargeted RNAi sequence (rectangles). Mitochondrial RNA synthesis was blocked by the addition of $250 \mathrm{ng} / \mathrm{ml}$ EtBr. Total RNA was prepared 0, 6, 12 and $24 \mathrm{~h}$ after addition of $\mathrm{EtBr}$ and used for quantitative real-time PCR. The results represent the mean \pm SEM from three independent experiments, each of which measured in triplicates

\section{Discussion}

We have shown that leucine deprivation influences the expression of the mitochondrial tRNA ${ }^{\mathrm{Leu}(\mathrm{CUN})}$ and the two nuclear-encoded genes LARS2 and PTCD1. The upregulation of the mitochondrial leucyl-tRNA synthetase LARS2 might enable a certain degree of mitochondrial translation when leucine is limited. A similar upregulation of several cytoplasmic aminoacyl-tRNA synthetases during amino acid deprivation is thought to preserve the synthesis of critical stress-responsive proteins (Sikalidis et al. 2011). Interestingly, LARS2 was also shown to be upregulated in cells carrying a $3243 \mathrm{~A}>\mathrm{G}$ point mutation in the gene for the mitochondrial tRNA ${ }^{\text {Leu(UUR), }}$, a mutation which is related to mitochondrial myopathy, encephalopathy, lactic acidosis, and stroke-like episodes (MELAS) (Munakata et al. 2005). This upregulation might partially compensate for the decreased efficiency of aminoacylation of tRNA $^{\text {Leu(UUR) }}$ (Munakata et al. 2005), since overexpression of LARS2 was later shown to correct for the mitochondrial dysfunction due to the MELAS mutation (Park et al. 2008; $\mathrm{Li}$ et al. 2010). The upregulation of LARS2 in MELAS suggests that there might be signalling from mitochondria to the nucleus when aminoacylation of mitochondrial tRNAs is insufficient.

We have identified PTCD1 as one of the genes whose mRNA steady-state levels are strongly increased under leucine deprivation. However, PTCD1 protein was significantly decreased in leucine-starved cells. This indicates that leucine starvation regulates PTCD1 expression mainly on the translational or post-translational level. Branched chain amino acids and, particularly, leucine are involved in the regulation of translation initiation in the cytoplasm, and leucine is also one of the eight amino acids, that regulate the breakdown of proteins (Fafournoux et al. 2000). Therefore, it is possible that PTCD1 protein in leucinestarved cells is decreased by a general inhibition of translation and increased proteolysis. The decrease of PTCD1 protein in our experiments was very fast, with a half-life of probably $<24 \mathrm{~h}$. This is very short compared to the estimated overall half-life of mitochondrial membrane proteins of 4.2 and 12.6 days for outer and inner membrane proteins, respectively in rat liver (Brunner and Neupert 1968). If PTCD1 indeed regulates mitochondrial translation by controlling leucine tRNA levels, it will affect the synthesis of the 13 mitochondrially encoded proteins, which are all localised in the inner mitochondrial membrane. A faster adaptation of the regulatory protein PTCD1 (half-life $<1$ day) by environmental stress compared to the affected proteins (overall half-life 12.6 days) is certainly required for a functional regulation. It is therefore possible, that the decrease of PTCD1 protein is indeed a part of the regulatory response to leucine deprivation.

Rackham et al. could show that PTCD1 overexpression decreases and PTCD1 knockdown increases mitochondrial tRNA $^{\text {Leu(CUN) }}$ and tRNA ${ }^{\text {Leu(UUR) }}$ steady-state levels (Rackham et al. 2009). Here, we show that the stability of the mitochondrial tRNA ${ }^{\mathrm{Leu}(\mathrm{CUN})}$ and tRNA $\mathrm{Leu}^{\mathrm{LuUR})}$ is increased by PTCD1. The mechanism by which PTCD1 


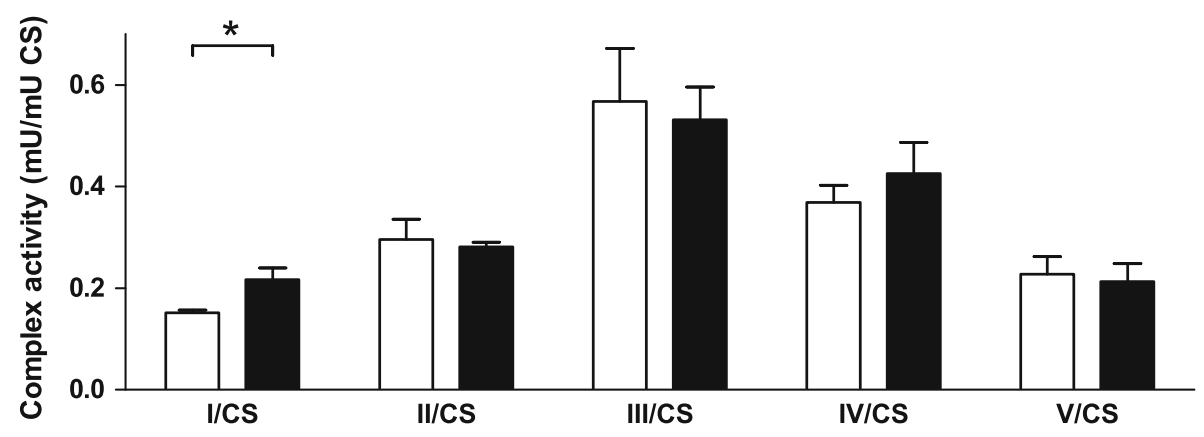

Fig. 5 Effect of PTCD1 knockdown on the enzymatic activities of the respiratory chain complexes in isolated mitochondria. Enzymatic activities of respiratory complexes and citrate synthase were measured in cells stably transfected with PTCD1 RNAi (black boxes) or non-targeted RNAi sequence (white boxes) by spectrophotometry and normalised to citrate synthase (CS). Mean \pm SEM of four independent experiments. ${ }^{*} p<0.05$ compared to non-targeted RNAi by a two-tailed $U$ test

Table 2 Leucine content of mitochondrially encoded proteins

\begin{tabular}{|c|c|c|c|c|c|c|}
\hline Name & Complex & $\mathrm{Nr}$ of aas ${ }^{\mathrm{a}}$ & $\mathrm{Nr}$ of tRNA ${ }^{\mathrm{Leu}(\mathrm{CUN})}$ & $\mathrm{Nr}$ of tRNA ${ }^{\text {Leu(UUR) }}$ & Leucine density $(\%)^{\mathrm{b}}$ & Leu(UUR) fraction $(\%)^{\circ}$ \\
\hline ND1 & CI & 318 & 57 & 6 & 19.8 & 9.5 \\
\hline ND2 & CI & 347 & 55 & 9 & 18.4 & 14.1 \\
\hline ND3 & CI & 115 & 18 & 10 & 24.3 & 35.7 \\
\hline ND4 & CI & 459 & 87 & 9 & 20.9 & 9.4 \\
\hline ND4L & CI & 98 & 22 & 1 & 23.5 & 4.3 \\
\hline ND5 & CI & 603 & 95 & 9 & 17.2 & 8.7 \\
\hline ND6 & CI & 174 & 3 & 14 & 9.8 & 82.4 \\
\hline CytB & CIII & 378 & 55 & 9 & 16.9 & 14.1 \\
\hline $\mathrm{COI}$ & CIV & 513 & 55 & 7 & 12.1 & 11.3 \\
\hline COII & CIV & 227 & 28 & 5 & 14.5 & 15.2 \\
\hline COIII & CIV & 260 & 31 & 3 & 13.1 & 8.8 \\
\hline ATP6 & $\mathrm{CV}$ & 226 & 39 & 5 & 19.5 & 11.4 \\
\hline ATP8 & $\mathrm{CV}$ & 68 & 8 & 2 & 14.7 & 20 \\
\hline
\end{tabular}

Complex I contains the subunits with the highest leucine density and leucine (UUR) fraction (marked in bold)

Adapted from Kirino et al. 2004

a Number of amino acids (aas)

b $100 \times\left(\mathrm{Leu}^{(\mathrm{CUN})}+\mathrm{Leu}^{(\mathrm{UUR})} /\right.$ total amino acids

c $100 \times \mathrm{Leu}^{(\mathrm{UUR})} /\left(\mathrm{Leu}^{(\mathrm{CUN})}+\mathrm{Leu}^{(\mathrm{UUR})}\right)$

regulates the steady-state levels of mitochondrial leucine tRNAs remains unknown. PTCD1 contains eight pentatricopeptide repeat (PPR) domains (Rackham et al. 2009). PPR domains are predicted to bind RNA and are included in numerous organellar proteins, where they are involved in posttranscriptional processes such as splicing, editing, processing and translation. It is assumed that most PPR proteins harbour no catalytic domain, but recruit a partner protein to the specific site on the RNA target (Lurin et al. 2004; Delannoy et al. 2007; Rackham and Filipovska 2012). Also on PTCD1, no nuclease domain could be identified by sequence comparison (Rackham et al. 2009). In addition to the function of PTCD1 protein to destabilise mitochondrial leucine tRNAs (Fig. 4) and decrease its steady-state levels (Rackham et al. 2009), PTCD1 was also shown to affect the $3^{\prime}$-end processing of tRNAs (Sanchez et al. 2011). However, PTCD1 knockdown seems not to lead to a loss of function, because we and others (Rackham et al. 2009; Sanchez et al. 2011) could show that respiration and activity of mitochondrial complexes were even higher in PTCD1 knockdown cells. Rackham et al. (2009) found increased activity of complex IV in PTCD1 knockdown cells in 143B osteosarcoma cells, but no significant difference in complex I activity. The discrepancy to our complex I result might be explained methodically: Rackham et al. measured complex I by mitochondrial respiration, and thereby included all five respiratory complexes in their measurement. In contrast, we measured the isolated activity of complex I. The finding of Rackham et al. that PTCD1 knockdown increased the steady-state levels of 
both complex I and IV proteins on Western blots indirectly supports our results (Rackham et al. 2009). Interestingly, complex I harbours the four proteins with the highest overall leucine content of all 13 mitochondrially encoded proteins. The leucine density of the complex I subunits ND1, ND3, ND4 and ND4L ranges between 19.8 and $24.3 \%$ of total amino acids, and hence is extremely high (Table 2). Since PTCD1 lowers the steady-state level and half-life of mitochondrial leucine tRNAs, it seems to be reasonable that lowering the PTCD1 protein level would affect mainly complex I proteins and activity.

Cytoplasmic tRNAs may be depleted by targeted destruction of tRNAs in response to stress such as amino acid starvation, oxidative stress, arsenite, UV irradiation and heat shock but not to apoptosis in general. This mechanism may enable cells to modulate translation in response to stress (Thompson et al. 2008; Rackham et al. 2009; Thompson and Parker 2009; Yamasaki et al. 2009). In mitochondria, the ribonuclease $\mathrm{L}$ was shown to be involved in the interferon-induced degradation of mitochondrial mRNAs, which may inhibit translation and lead to apoptosis (Le Roy et al. 2007). PTCD1 was the first protein postulated to be involved in the targeted destruction of tRNA in an organelle (Rackham et al. 2009). Interestingly, our results indicate that decreased PTCD1 protein levels stabilised mitochondrial leucine tRNAs in HepG2 cells. Under leucine starvation PTCD1 protein is decreased. This may contribute to maintain adequate mitochondrial translation by stabilising mitochondrial tRNAs. PTCD1 might, therefore, play a central role in adaptation of mitochondria to amino acid deprivation.

Conflict of interest None declared.

\section{References}

Averous J, Bruhat A, Jousse C, Carraro V, Thiel G et al (2004) Induction of $\mathrm{CHOP}$ expression by amino acid limitation requires both ATF4 expression and ATF2 phosphorylation. J Biol Chem 279(7):5288-5297

Brunner G, Neupert W (1968) Turnover of outer and inner membrane proteins of rat liver mitochondria. FEBS Lett 1(3):153-155

Chaveroux C, Lambert-Langlais S, Cherasse Y, Averous J, Parry L et al (2010) Molecular mechanisms involved in the adaptation to amino acid limitation in mammals. Biochimie 92(7):736-745

Delannoy E, Stanley WA, Bond CS, Small ID (2007) Pentatricopeptide repeat (PPR) proteins as sequence-specificity factors in posttranscriptional processes in organelles. Biochem Soc Trans 35(Pt 6): $1643-1647$

Doering CB, Danner DJ (2000) Amino acid deprivation induces translation of branched-chain alpha-ketoacid dehydrogenase kinase. Am J Physiol Cell Physiol 279(5):C1587-C1594

Fafournoux P, Bruhat A, Jousse C (2000) Amino acid regulation of gene expression. Biochem J 351(Pt 1):1-12

Harding HP, Zhang Y, Zeng H, Novoa I, Lu PD et al (2003) An integrated stress response regulates amino acid metabolism and resistance to oxidative stress. Mol Cell 11(3):619-633
Harris RA, Kobayashi R, Murakami T, Shimomura Y (2001) Regulation of branched-chain alpha-keto acid dehydrogenase kinase expression in rat liver. J Nutr 131(3):841S-845S

Jackson CB, Nuoffer JM, Hahn D, Prokisch H, Haberberger B, Gautschi M, Häberli A, Gallati S, Schaller A (2014) Mutations in SDHD lead to autosomal recessive encephalomyopathy and isolated mitochondrial complex II deficiency. J Med Genet 51(3):170-175. doi:10.1136/jmedgenet-2013-101932

Kirino Y, Yasukawa T, Ohta S, Akira S, Ishihara K, Watanabe K, Suzuki T (2004) Codon-specific translational defect caused by a wobble modification deficiency in mutant tRNA from a human mitochondrial disease. PNAS 101(42):15070-15075

Kristensen AR, Schandorff S, Hoyer-Hansen M, Nielsen MO, Jaattela $M$ et al (2008) Ordered organelle degradation during starvation-induced autophagy. Mol Cell Proteomics 7(12): 2419-2428

Le Roy F, Silhol M, Salehzada T, Bisbal C (2007) Regulation of mitochondrial mRNA stability by RNase L is translationdependent and controls IFNalpha-induced apoptosis. Cell Death Differ 14(8):1406-1413

Lee JI, Dominy JE Jr, Sikalidis AK, Hirschberger LL, Wang W et al (2008) HepG2/C3A cells respond to cysteine deprivation by induction of the amino acid deprivation/integrated stress response pathway. Physiol Genomics 33(2):218-229

Leung-Pineda V, Pan Y, Chen H, Kilberg MS (2004) Induction of p21 and p27 expression by amino acid deprivation of HepG2 human hepatoma cells involves mRNA stabilization. Biochem J 379(Pt 1):79-88

Li M, Schonberg A, Schaefer M, Schroeder R, Nasidze I et al (2010) Detecting heteroplasmy from high-throughput sequencing of complete human mitochondrial DNA genomes. Am J Hum Genet 87(2):237-249

Lurin C, Andres C, Aubourg S, Bellaoui M, Bitton F et al (2004) Genome-wide analysis of Arabidopsis pentatricopeptide repeat proteins reveals their essential role in organelle biogenesis. Plant Cell 16(8):2089-2103

Munakata K, Iwamoto K, Bundo M, Kato T (2005) Mitochondrial DNA $3243 \mathrm{~A}>\mathrm{G}$ mutation and increased expression of LARS2 gene in the brains of patients with bipolar disorder and schizophrenia. Biol Psychiatry 57(5):525-532

Paillusson A, Hirschi N, Vallan C, Azzalin CM, Muhlemann O (2005) A GFP-based reporter system to monitor nonsense-mediated mRNA decay. Nucleic Acids Res 33(6):e54

Park H, Davidson E, King MP (2008) Overexpressed mitochondrial leucyl-tRNA synthetase suppresses the A3243G mutation in the mitochondrial tRNA(Leu(UUR)) gene. RNA 14(11):2407-2416

Rackham O, Davies SM, Shearwood AM, Hamilton KL, Whelan J et al (2009) Pentatricopeptide repeat domain protein 1 lowers the levels of mitochondrial leucine tRNAs in cells. Nucleic Acids Res 37(17):5859-5867

Rackham O, Filipovska A (2012) The role of mammalian PPR domain proteins in the regulation of mitochondrial gene expression. Biochim Biophys Acta 1819(9-10):1008-1016

Rambold AS, Kostelecky B, Elia N, Lippincott-Schwartz J (2011) Tubular network formation protects mitochondria from autophagosomal degradation during nutrient starvation. Proc Natl Acad Sci USA 108(25):10190-10195

Sanchez MI, Mercer TR, Davies SM, Shearwood AM, Nygard KK et al (2011) RNA processing in human mitochondria. Cell Cycle 10(17):2904-2916

Schaller A, Hahn D, Jackson CB, Kern I, Chardot C et al (2011) Molecular and biochemical characterisation of a novel mutation in POLG associated with Alpers syndrome. BMC Neurol 11:4

Sikalidis AK, Lee JI, Stipanuk MH (2011) Gene expression and integrated stress response in HepG2/C3A cells cultured in amino acid deficient medium. Amino Acids 41(1):159-171 
Siu F, Bain PJ, LeBlanc-Chaffin R, Chen H, Kilberg MS (2002) ATF4 is a mediator of the nutrient-sensing response pathway that activates the human asparagine synthetase gene. J Biol Chem 277(27):24120-24127

Sun X, Zemel MB (2009) Leucine modulation of mitochondrial mass and oxygen consumption in skeletal muscle cells and adipocytes. Nutr Metab (Lond) 6:26

Thompson DM, Lu C, Green PJ, Parker R (2008) tRNA cleavage is a conserved response to oxidative stress in eukaryotes. RNA 14(10):2095-2103
Thompson DM, Parker R (2009) Stressing out over tRNA cleavage. Cell 138(2):215-219

Yamasaki S, Ivanov P, Hu GF, Anderson P (2009) Angiogenin cleaves tRNA and promotes stress-induced translational repression. J Cell Biol 185(1):35-42

Yasukawa T, Hino N, Suzuki T, Watanabe K, Ueda T et al (2000) A pathogenic point mutation reduces stability of mitochondrial mutant tRNA(Ile). Nucleic Acids Res 28(19):3779-3784 